\title{
Конкуренция за внимание пространственных модуляций градиентов яркости
}

\author{
Виталий В. Бабенко“, Денис В. Явна \\ Южный федеральный университет, г. Ростов-на-Дону, Российская Федерация \\ *E-mail: babenko@sfedu.ru
}

\begin{abstract}
Аннотация
Ввеление. Преаттентивный этап зрительной обработки вкАючает стаАию вылеления и кодирования градиентов яркости, а также сталию пространственного объединения этой инорормации. Первая операция реализуется зрительными фрильтрами первого порядка (стриарными нейронами), вторая - фрильтрами второго порялка (экстрастриарными нейронами). Фильтры второго порядка пропускают об^асти изображения, характеризующиеся наличием пространственных модуляций контраста, ориентации и пространственной частоты (размерности модуляции) и могут играть роль ворот внимания. Целью исслеАования является установление приоритетов среАи моАуляций в конкуренции за внимание.
\end{abstract}

Методы. Все стимулы состояли из трех изображений одного и того же объекта. Эти изображения были сорормированы из областей исходного объекта, содержащих модуляции контраста (одно изображение), ориентации (второе изображение) и пространственной частоты (третье изображение). Сами эти области предварительно вылелялись из объекта с помощью разработанной модели фрильтров второго порядка. В первом эксперименте за внимание конкурировали изображения, сорормированные из модуляций разной размерности. Во втором - изображения одной размерности, отличающиеся по пространственной частоте. Привлечение внимания определялось путем регистрации Авижений глаз.

Результаты. Обнаружено, что в конкуренции межАу размерностями преимущество имели МоАуляции контраста и ориентации. КогАа выбор осуществАя^ся межАУ изображениями одной размерности, побежАали изображения, сорормированные из «среАних» пространственных частот.

Обсужаение результатов. Впервые получены данные о приоритетах срели модуляций в конкуренции за внимание. Установлено, что чем выше среАний выхо ффильтров второго порялка, фрормирующих тестовое изображение, тем выше вероятность того, что это изображение привлечет внимание испытуемого. Заключение. Аелается вывоА, что фрильтры второго порядка могут играть роль ворот внимания, при этом преимущество в конкуренции за внимание в залаче обнаружения получают фрильтры с большей величиной сигнала на своем выхоле. 


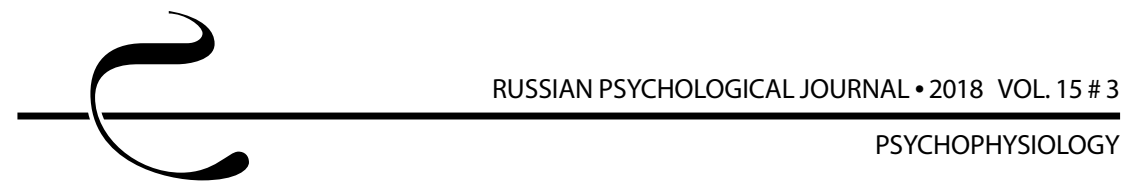

В приложении описываются модели зрительных механизмов второго порядка, использованные при подготовке стимульного материала.

\title{
КАючевые слова
}

градиенты яркости, пространственная модуляция, зрительные фрильтры, контраст, ориентация, пространственная частота, вылеление огибающей, Авижения глаз, управление вниманием, молелирование

\section{Основные положения}

- среди модуляций разной размерности более приоритетными в конкуренции за внимание являются моАуляции контраста и ориентации;

- среАи модуляций оАной размерности более приоритетными в конкуренции за внимание являются модуляции со «средней» частотой несущей;

- при решении залачи зрительного обнаружения преимущество в конкуренции за внимание получают фрильтры второго порядка с наибольшей величиной сигнала на своем вхоле.

\section{Для цитирования}

Бабенко В.В., Явна Д.В. Конкуренция за внимание пространственных модуляций градиентов яркости // Российский психологический журнал. 2018. Т. 15, № 3. С. 160-189. DOI: 10.21702/rpj.2018.3.8

Материалы статьи получены 13.02.2018

UDC 159.938

DOI: $10.21702 /$ rpj.2018.3.8

\section{Competition for Attention Among Spatial Modulations of Brightness Gradients}

\section{Vitalii V. Babenko*, Denis V. Yavna}

Southern Federal University, Rostov-on-Don, Russian Federation

${ }^{*}$ Corresponding author. E-mail: babenko@sfedu.ru

\begin{abstract}
Introduction. The pre-attentive stage of visual processing includes the stage of selection and coding of brightness gradients, and the stage of spatial integration of this information. The first operation is realized by the first-order visual filters (striatal neurons), the second one - by the second-order visual filters (extrastriatal neurons). The second-order filters transmit image areas with spatial modulations of contrast, orientation, and spatial frequency (modulation dimensions) and can function as attention gates. This investigation is aimed at establishing priorities among the modulations in their competition for attention.
\end{abstract}


Methods. All the stimuli in the study represented three images of the same object. These images were constructed from the areas of the original object and contained modulations of a) contrast (first image), b) orientation (second image), and c) spatial frequency (third image). These areas were in advance isolated from the object by means of the model of the second-order filters developed by the authors. In the first experiment, images composed of different in dimension modulations competed for participants' attention. In the second experiment, the authors used images of the same dimension, but different in their spatial frequency. Attention focus was determined by recording eye movements.

Results. The findings suggest that the modulations of contrast and orientation had an advantage in the competition for attention among dimensions. When the choice was made between the images of the same dimension, the images formed from the middle-range spatial frequencies had the advantage in the competition for attention.

Discussion. The present study is the first to address the priorities among the modulations in competition for attention. Higher average rates of output signals in the second-order filters that form the test image increase the probability that this image will attract participants' attention.

Conclusion. The second-order filters can play the role of attention gates. In addition, the filters with higher rates of their output signals have an advantage in competing for attention in visual search. The Appendix describes the models of the second-order visual mechanisms, which the authors used for preparing the study stimuli.

\section{Keywords}

brightness gradients, spatial modulation, visual filters, contrast, orientation, spatial frequency, envelope selection, eye movements, attention control, simulation

\section{Highlights}

- Among the modulations of different dimensions, modulations of contrast and orientation are of higher priority in the competition for attention.

- Among the modulations of the same dimension, modulation with the average carrier frequency is of higher priority in the competition for attention.

- The second-order filters with the highest rates of output signals have an advantage in the competition for attention in visual search.

\section{For citation}

Babenko V.V., Yavna D.V. Competition for Attention Among Spatial Modulations of Brightness Gradients. Rossiiskii psikhologicheskii zhurnal - Russian Psychological Journal, 2018, V. 15, no. 3, pp. 160-189 (in Russian). DOI: 10.21702/rpj.2018.3.8 


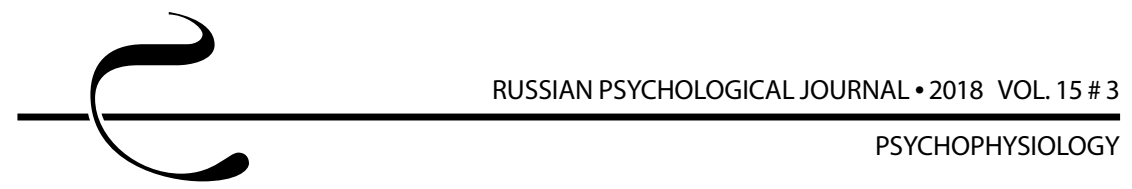

\section{Введение}

Пытаясь ответить на вопрос о механизмах управления зрительным вниманием, многие авторы использовали данные о движениях глаз $[1,2,3,4]$. Один из важных выводов этих исследований состоит в том, что начальные фиксации определяются главным образом физическими параметрами элементов сцены, а последующие - их семантикой $[5,6,7]$.

Когда речь идет об управлении вниманием «снизу», следует отдавать себе отчет в том, что внимание притягивается не объектами внешнего мира, а результатами их преаттентивной обработки. Такими результатами являются, в частности, выделяемые стриарными нейронами градиенты яркости $[8,9]$. Исследования показали, что в конкуренции за внимание побеждают те градиенты, которые имеют, например, больший контраст [10]. Были определены и другие параметры перепадов яркости, которые влияют на направленность внимания $[11,12,13]$.

Однако в такого рода экспериментах использовались, как правило, искусственно созданные стимулы. Когда же исследователи переходили к реальным сценам, ни одна из моделей управления вниманием не могла с достаточной точностью предсказать распределение фиксаций взора $[14,15,16]$. Это можно объяснить тем, что внимание могут притягивать не только локальные фрагменты изображения, но и более сложные конструкции. Но какая еще информация может присутствовать на выходе преаттентивной обработки, кроме локальных признаков?

В последние десятилетия были предсказаны, обнаружены и подробно изучены так называемые зрительные фильтры 2-го порядка (Ф2П), которые объединяют выходы простых стриарных нейронов (фильтров 1-го порядка) $[17,18,19]$. О наличии в мозге определенного нейрофизиологического субстрата, обеспечивающего обнаружение и анализ пространственных модуляций первичных зрительных признаков, свидетельствуют, в том числе, и данные, полученные Д.В. Явна с соавт. [20, 21].

Ф2П обнаруживают пространственные модуляции градиентов яркости. И именно такие модуляции делают видимый нами мир бесконечно разнообразным (также как модуляции тонов делают разнообразным звуковой мир). Вполне логично предположить, что выходы Ф2П также конкурируют за внимание. Однако данная проблема находится лишь в начале своего решения. Известно, что пространственные модуляции могут иметь разную размерность: это могут быть изменения контраста, ориентации или пространственной частоты градиентов яркости. Кроме того, эти модуляции выделяются зрительной системой человека в шести разных диапазонах пространственных частот (по числу зрительных путей). А это также может быть фактором, определяющим приоритетность тех или иных областей изображения. 
Цель нашего исследования состояла в том, чтобы определить, как при конкуренции за внимание распределяются приоритеты среди модуляций разной размерности одинаковой пространственной частоты и среди модуляций разной пространственной частоты в рамках одной размерности. В первом эксперименте за внимание испытуемого конкурировали три изображения одного и того же объекта, сформированные из его областей с модуляциями контраста, ориентации и пространственной частоты. Во втором эксперименте конкурирующие изображения были сформированы из модуляций одной размерности, но отличались пространственной частотой.

О привлечении внимания к тестовым изображениям судили по результатам регистрации движений глаз. Появляясь в поле зрения, новый объект автоматически привлекает к себе внимание, вызывая перевод взора. Если одновременно появляется несколько объектов, то возникает ситуация их конкуренции за внимание. Учитывая, что направленность взора может зависеть от множества факторов, а сам глазодвигательный акт складывается фактически в ходе самого процесса зрительного восприятия [22], наша задача состояла в том, чтобы выделить из этой сложной совокупности взаимосвязанных процессов самый начальный и наиболее автоматизированный этап - первый перевод взора [23].

\section{Методы}

\section{Испытуемые}

В каждом из экспериментов приняли участие по 20 испытуемых обоего пола (в первом эксперименте - 9 мужчин и 11 женщин, во втором эксперименте - по 10 человек) в возрасте от 20 до 32 лет. Все имели нормальное или скорректированное до нормы зрение. Все убеждались в безопасности процедуры и участвовали в исследовании добровольно. Исследование проводилось с соблюдением этических норм, в соответствии с Хельсинским соглашением, и одобрено местной комиссией по этике.

\section{Annapamypa}

Для регистрации движений глаз использовался трекер RED-m производства SensoMotoric Instruments $\mathrm{GmbH}$ (SMI), подключенный к персональному компьютеру с процессором Intel Core i5-4460 и графическим адаптером Nvidia GT 730, работающему под управлением OC Windows 10. Стимулы демонстрировались на дисплее ViewSonic VX2263Smhl с отключенной функцией динамического контраста и пиксельным разрешением 1920 на 1080 точек. Процедура предъявления была организована в программе SMI Experiment Center 3.6. 


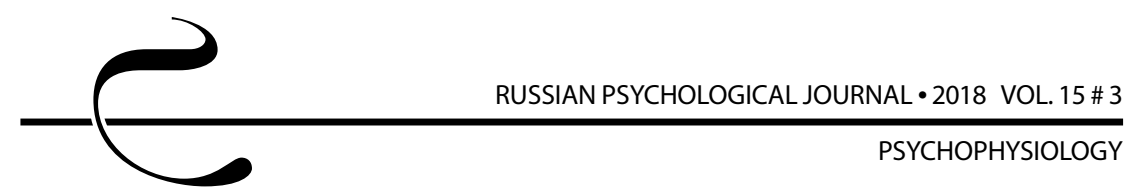

\section{Стимулы}

Для подготовки стимулов использовались оцифрованные полутоновые фотографии 100 объектов разных категорий (естественной и искусственной природы). Изображения были выровнены по средней яркости и диапазону контраста, приведены к единому размеру, помещены на однородный фон и подвергнуты обработке с помощью разработанной нами компьютерной модели зрительных фильтров второго порядка, избирательных к размерности модуляции (см. приложение). Целью обработки было выделить в этих объектах области с модуляциями контраста, ориентации и пространственной частоты в трех диапазонах пространственных частот (1-4, 2-8 и 4-16 цикл./град.). Эти диапазоны соответствуют частотным настройкам зрительных путей человека в нижней половине спектра [24].

Затем исходные объекты «восстанавливались» из областей, содержащих модуляции одной определенной размерности. В частности, в единое изображение объединялись фрагменты исходного объекта, содержащие модуляции контраста. Для эксперимента 1 это были области, выделенные во всех трех диапазонах пространственных частот. В результате получали «восстановленное» изображение с полосой пространственных частот порядка 4 октав (1-16 цикл./град.). Для эксперимента 2 по отдельности объединялись области, выделенные в каждом из частотных диапазонов. Результатом были 3 изображения, восстановленных из областей с модуляциями контраста, но отличающихся пиковой частотой несущей $(2,4$ и 8 цикл./град.) при ширине полос 2 октавы. Таким же способом объединялись фрагменты, содержащие модуляции ориентации и модуляции пространственной частоты. В результате из каждого исходного объекта было сформировано 12 восстановленных изображений: 3 - для первого эксперимента и 9 - для второго (таблица 1).

После того, как были созданы восстановленные изображения, переходили к формированию стимулов. Каждый стимул представлял собой однородное серое поле яркостью $30 \mathrm{kд} / \mathrm{M}^{2}$, на которое помещались три восстановленных изображения одного и того же объекта. Все они имели размер по 3,7 угл. град. и помещались в случайной позиции на условной окружности радиусом 5,75 угл. град. Расстояние между ними было всегда одинаковым. Стимулы для эксперимента 1 содержали 3 изображения разной размерности, но одинакового частотного наполнения (рисунок 1). Стимулы для эксперимента 2 включали 3 изображения одной размерности, но разной пространственной частоты (рисунок 2). В таблице 1 в рамки заключены изображения, которые объединялись в единый тестовый стимул. В общей сложности для эксперимента 1 было сформировано 100 стимулов, для эксперимента 2 - 300 (по 100 стимулов для каждой серии). 
Таблица 1. Тестовые изображения, сфформированные из одного исходного объекта

Table 1. Test images constructed from the original object

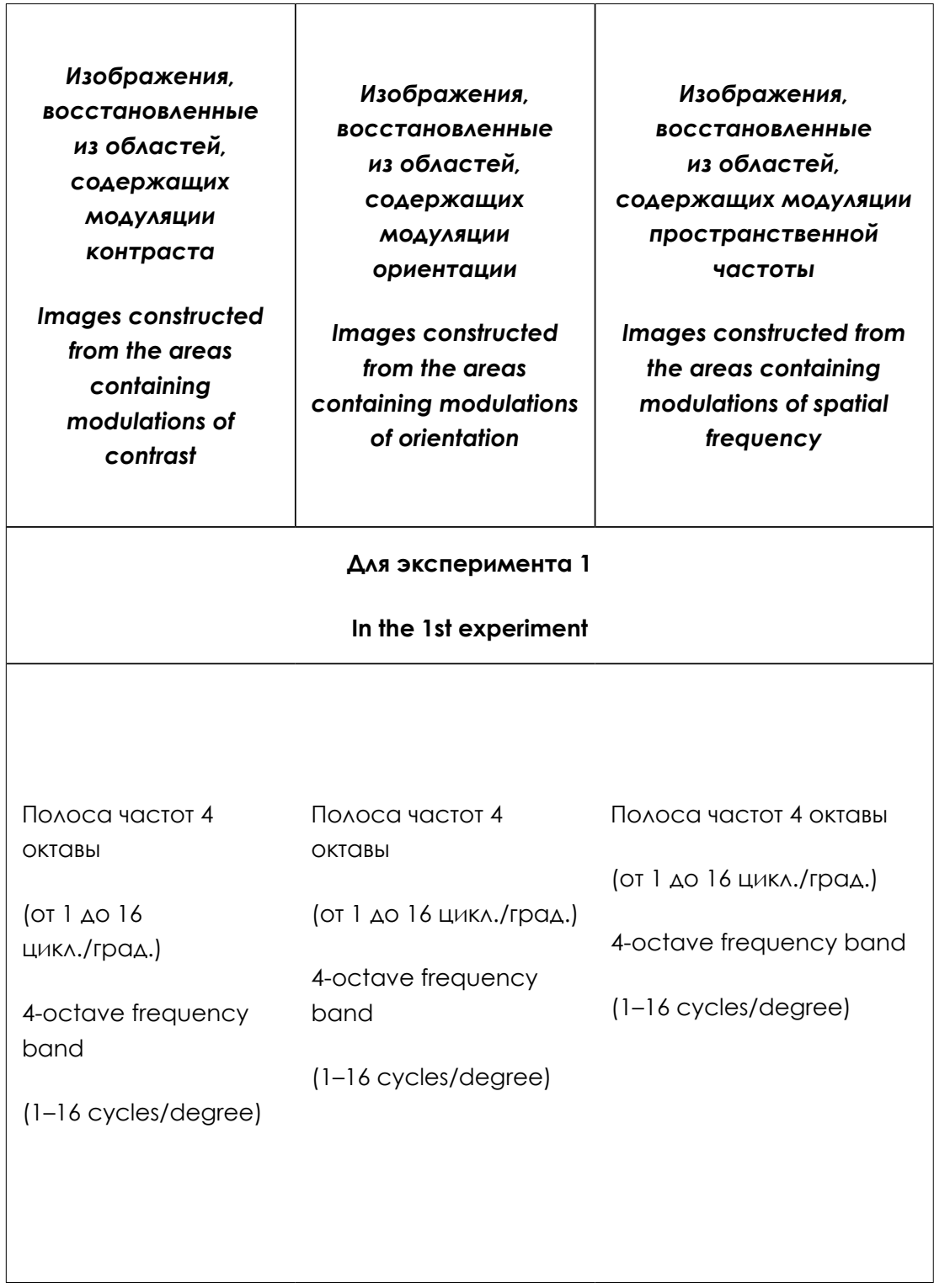




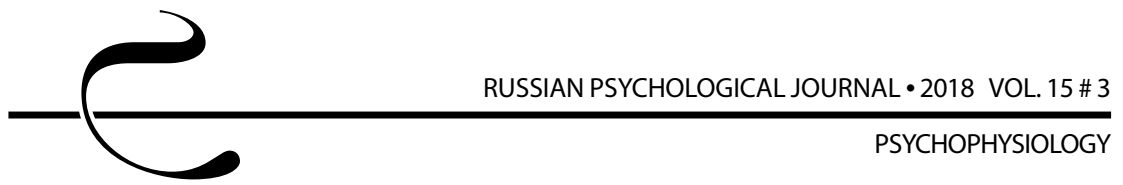

\begin{tabular}{|c|c|c|}
\hline \multicolumn{3}{|c|}{$\begin{array}{l}\text { А^я эксперимента } 2 \\
\text { In the 2nd experiment }\end{array}$} \\
\hline $\begin{array}{l}\text { Серия } 1 \\
\text { Series } 1\end{array}$ & $\begin{array}{l}\text { Серия } 2 \\
\text { Series } 2\end{array}$ & $\begin{array}{l}\text { Серия } 3 \\
\text { Series } 3\end{array}$ \\
\hline $\begin{array}{l}\text { Полоса частот } 2 \\
\text { октавы } \\
\text { (от } 1 \text { до } 4 \text { цикл./грал.) } \\
\text { 2-octave frequency } \\
\text { band } \\
\text { (1-4 cycles/degree) }\end{array}$ & $\begin{array}{l}\text { Полоса частот } 2 \\
\text { октавы } \\
\text { (от } 1 \text { Ао } 4 \text { цикл./грал.) } \\
\text { 2-octave frequency } \\
\text { band } \\
\text { (1-4 cycles/degree) }\end{array}$ & $\begin{array}{l}\text { Полоса частот } 2 \text { октавы } \\
\text { (от } 1 \text { Ао } 4 \text { цикл./гра..) } \\
\text { 2-octave frequency band } \\
\text { (1-4 cycles/degree) }\end{array}$ \\
\hline $\begin{array}{l}\text { Полоса частот } 2 \\
\text { октавы } \\
\text { (от } 2 \text { Ао } 8 \text { цикл./грал.) } \\
\text { 2-octave frequency } \\
\text { band } \\
\text { (2-8 cycles/degree) }\end{array}$ & $\begin{array}{l}\text { Полоса частот } 2 \\
\text { октавы } \\
\text { (от } 2 \text { Ао } 8 \text { цикл./грал.) } \\
\text { 2-octave frequency } \\
\text { band } \\
\text { (2-8 cycles/degree) }\end{array}$ & $\begin{array}{l}\text { Полоса частот } 2 \text { октавы } \\
\text { (от } 2 \text { Ао } 8 \text { цикл./граА.) } \\
\text { 2-octave frequency band } \\
\text { (2-8 cycles/degree) }\end{array}$ \\
\hline $\begin{array}{l}\text { Полоса частот } 2 \\
\text { октавы } \\
\text { (от } 4 \text { Ао } 16 \\
\text { цикл./грал.) } \\
\text { 2-octave frequency } \\
\text { band } \\
\text { (4-16 cycles/degree) }\end{array}$ & $\begin{array}{l}\text { Полоса частот } 2 \\
\text { октавы } \\
\text { (от } 4 \text { Ао } 16 \text { цикл./грал.) } \\
\text { 2-octave frequency } \\
\text { band } \\
\text { (4-16 cycles/degree) }\end{array}$ & $\begin{array}{l}\text { Полоса частот } 2 \text { октавы } \\
\text { (от } 4 \text { Ао } 16 \text { цикл./град.) } \\
\text { 2-octave frequency band } \\
\text { (4-16 cycles/degree) }\end{array}$ \\
\hline
\end{tabular}




\section{Прочедура}

Перед началом эксперимента испытуемому зачитывалась инструкция, которая разъясняла порядок предъявления стимулов и стоящую перед ним задачу. Перед предъявлением стимула испытуемый должен был направить взор на черное перекрестье в центре экрана, заполненного однородным серым фоном яркостью 30 кд/м².

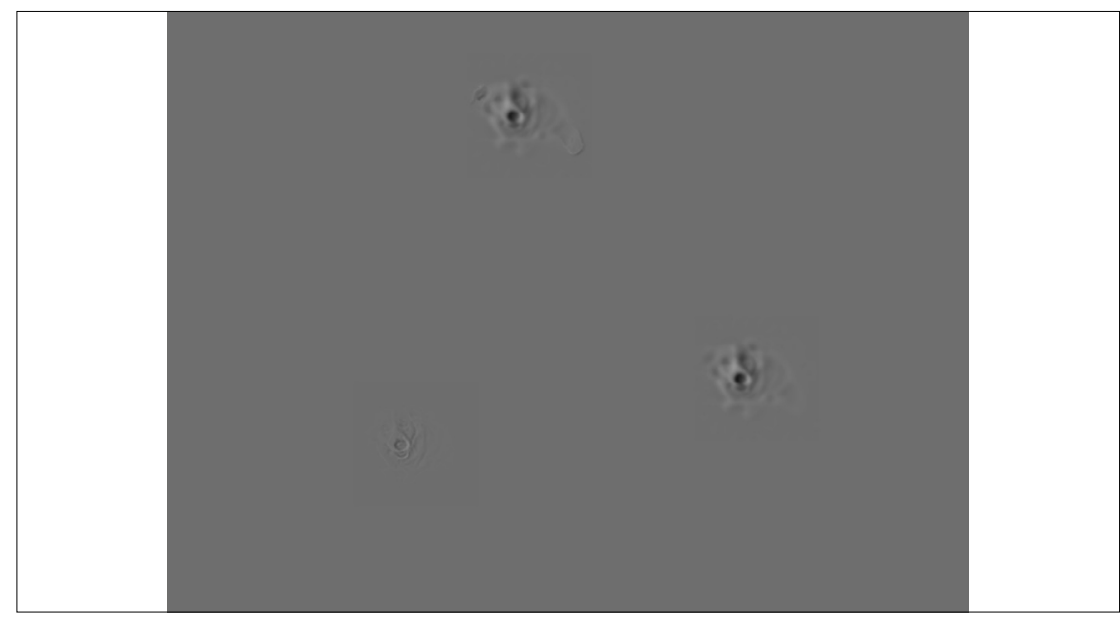

Рисунок 1. Пример стимула, использованного в эксперименте 1. Изображения собаки, сорормированные из областей с модуляциями разной размерности

Figure 1. An example of a stimulus used in the 1st experiment: Images of a dog composed of different in dimension modulations

Запуск каждого очередного стимула осуществлялся экспериментатором: поле с перекрестьем сменялось полем той же яркости с расположенными на нем тремя изображениями.

После их появления испытуемый мог свободно переводить взор. Время предъявления стимулов было ограничено одной секундой. Задача испытуемого состояла в определении локализации показанных изображений. Свой ответ он сообщал, ориентируясь на условный циферблат часов. В каждой пробе регистрировалась траектория движений глаз.

В целях повышения точности регистрации движений глаз голова испытуемого располагалась в лобно-подбородном упоре на расстоянии 60 см от экрана монитора. Перед началом эксперимента проводилась калибровка регистратора с помощью встроенной программы. Регистрация глазных движений осуществлялась в бинокулярном режиме с частотой 60 Гц. 

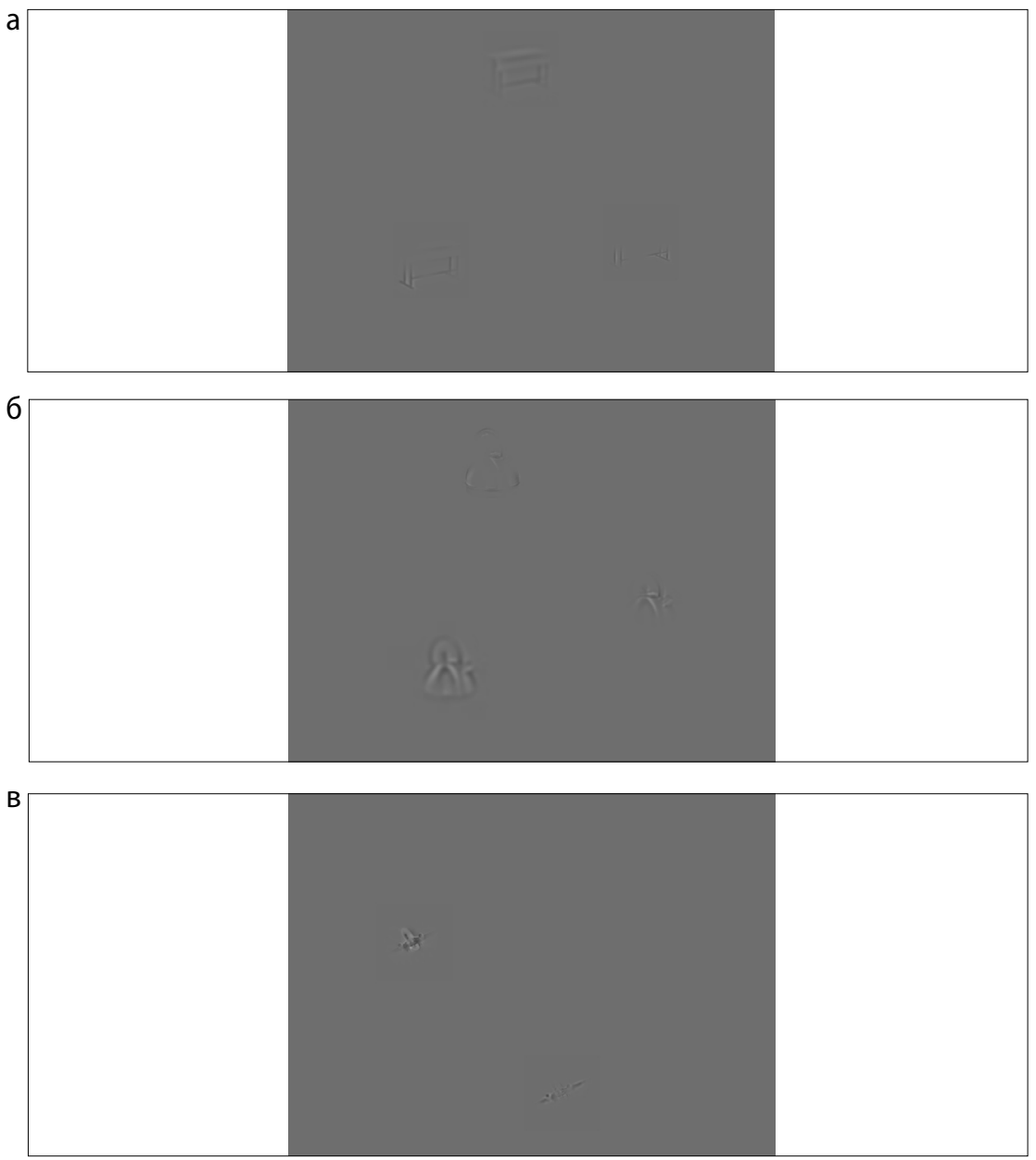

Рисунок 2. Примеры стимулов, использованных в эксперименте 2

Условные обозначения: $а$ - изображения (nарта) сформированы из областей с модулячиями контраста разной пространственной частоты; б - изображения (чайник) сформированы из областей с модуляциями ориентации; в- изображения (самолет) сформированы из областей с модулячиями пространственной частоты.

Figure 2. Examples of stimuli used in the 2 nd experiment

Legend: $a$-images (a school desk) constructed from the areas with the modulations of contrast differing in their spatial frequency; $b$-images (a kettle) constructed from the areas with the modulations of orientation; c-images (an airplane) constructed from the areas with the modulations of spatial frequency. 


\section{Обработка результатов}

Анализ фиксаций взора осуществлялся в отсроченном режиме с использованием программы SMI IDF Event Detector 3.0.20. Применялись настройки, принятые по умолчанию: время фиксации не должно быть менее 80 мс, максимальная дисперсия - 100 пикселей (примерно 2,34 угл. град.). Данные о фиксациях визуализировались средствами языка оболочки bash и пакета графических утилит ImageMagick.

Определялось, на какое из предъявленных изображений осуществлялся первый перевод взора. Затем для каждого испытуемого вычислялся процент первых фиксаций, направленных на каждое из конкурирующих изображений. Для определения статистической значимости обнаруженных различий использовался G-критерий согласия, принадлежащий к группе тестов отношения правдоподобия. Данный критерий является более универсальной альтернативой $\chi^{2}$, т. к. обладает свойством аддитивности и позволяет объединять результаты, полученные на разных испытуемых [25].

\section{Результаты}

\section{Эксперимент 1}

В первом эксперименте мы исследовали приоритетность пространственных модуляций разной размерности в конкуренции за внимание. При этом восстановленные изображения не отличались по пространственной частоте.

Оказалось, что первая фиксация взора могла быть направлена на любое из показанных изображений. То есть за внимание конкурировали все использованные модуляции. Однако вероятность, с которой каждая из них притягивала внимание, была разной. Обобщение данных по всем испытуемым показало, что в $36 \%$ случаев первый перевод взора осуществлялся на изображение, сформированное из модуляций контраста. В 35 \% случаев приоритет оставался за модуляциями ориентации. И лишь в $29 \%$ случаев внимание испытуемого привлекала модуляция пространственной частоты. Результаты, приведенные в таблице 2, указывают на то, что обнаруженные различия не случайны.

Оказалось, что модуляции пространственной частоты реже становились целью первой фиксации, чем модуляции контраста и ориентации. В то же время и модуляции контраста, и модуляции ориентации привлекали внимание испытуемых примерно с равной вероятностью.

Таким образом, результаты эксперимента 1 свидетельствуют о том, что внимание могут привлекать пространственные модуляции любой размерности. Однако модуляции контраста и ориентации более эффективны в конкуренции за внимание, чем модуляции пространственной частоты. 
Таблица 2. Результаты статистического сравнения вероятностей первой фиксации взора на изображениях, сорормированных из модуляций контраста, ориентации и пространственной частоты

Table 2. Results of statistical comparing the probabilities of participants' first fixation on the images composed of the modulations of contrast, orientation, and spatial frequency

\begin{tabular}{|c|c|c|}
\hline $\begin{array}{l}\text { Пары сравнения } \\
\text { Comparing pairs }\end{array}$ & $\begin{array}{c}\text { G-критерий } \\
\text { G-criterion }\end{array}$ & $\begin{array}{c}\text { р-значение } \\
\text { (Авустороннее) } \\
\text { p-value (two-tailed) }\end{array}$ \\
\hline $\begin{array}{l}\text { Модуляция контраста- } \\
\text { модуляция частоты } \\
\text { Modulation of } \\
\text { contrast-modulation of } \\
\text { spatial frequency }\end{array}$ & 12,9520 & 0,0003 \\
\hline $\begin{array}{l}\text { Модуляция контраста - } \\
\text { модуляция ориентации } \\
\text { Modulation of contrast- } \\
\text { modulation of orientation }\end{array}$ & 0,0711 & 0,7897 \\
\hline $\begin{array}{l}\text { Модуляция ориентации - } \\
\text { модуляция частоты } \\
\text { Modulation of } \\
\text { orientation - modulation of } \\
\text { spatial frequency }\end{array}$ & 11,1054 & 0,0009 \\
\hline
\end{tabular}

Примечание: здесь и далее жирным шрифтом выделены статистически значимые отличия. Note: here and below statistically significant differences are marked in bold.

\section{Эксперимент 2}

В этом эксперименте мы сравнивали способность демонстрируемых изображений привлекать внимание, когда они были сформированы из модуляций одной размерности, но отличались пространственной частотой. Эксперимент состоял из трех серий: в первой использовались модуляции контраста, во второй - модуляции ориентации, в третьей - модуляции пространственной частоты. Очередность серий менялась от испытуемого к испытуемому случайным образом. В остальном эксперимент повторял предыдущий. 
психоФизиология

\section{Серия 1.}

Оказалось, что когда стимулы были сформированы из модуляций контраста, самые низкочастотные из использованных изображений (пиковая частота 2 цикл./град.) становились целью первой фиксации в 28 \% случаев. Изображения с более высокой пространственной частотой (4 цикл./град.) притягивали взор в 29 \% случаев, а самые высокочастотные из использованных (8 цикл./град.) - в $22 \%$. Можно обратить внимание, что сумма всех указанных значений меньше $100 \%$. Это объясняется тем, что в некоторых пробах взгляд испытуемого не фиксировался на каком-либо из показанных объектов. Результаты статистического сравнения данных, полученных для изображений, сформированных из модуляций контраста разной пространственной частоты, показаны в таблице 3.

Приведенные в таблице 3 данные свидетельствуют о том, что когда все три изображения в стимулах были сформированы из модуляций контраста, самые высокочастотные существенно (статистически значимо) уступали другим в конкуренции за внимание. Вместе с тем два других изображения привлекали внимание испытуемых с равной вероятностью.

Таблица 3. Результаты статистического сравнения вероятностей первой фриксации взора на изображениях разной пространственной частоты, сорормированных Из МоАуляций контраста

Table 3. Results of statistical comparing the probabilities of participants' first fixation on the images composed of the modulations of contrast differing in their spatial frequency

\begin{tabular}{|c|c|c|}
\hline $\begin{array}{l}\text { Пары сравнения } \\
\text { Comparing pairs }\end{array}$ & $\begin{array}{c}\text { G-критерий } \\
\text { G-criterion }\end{array}$ & $\begin{array}{c}\text { р-значение } \\
\text { (Авустороннее) } \\
\text { p-value (two-tailed) }\end{array}$ \\
\hline $\begin{array}{l}8 \text { цикл./изобр. - } 16 \text { цикл./изобр. } \\
8 \text { cycles/image - } 16 \text { cycles/image }\end{array}$ & 0,0009 & 0,9764 \\
\hline $\begin{array}{l}8 \text { цикл./изобр. - } 32 \text { цикл./изобр. } \\
8 \text { cycles/image - } 32 \text { cycles/image }\end{array}$ & 19,8873 & 0,0000 \\
\hline $\begin{array}{l}16 \text { цикл./изобр. - } 32 \text { цикл./изобр. } \\
16 \text { cycles/image - } 32 \text { cycles/image }\end{array}$ & 20,1512 & 0,0000 \\
\hline
\end{tabular}




\section{Серия 2.}

Когда демонстрировались стимулы, в которых все три изображения были сформированы из модуляций ориентации, вероятность первой фиксации распределилась между изображениями разной пространственной частоты следующим образом. Самые низкочастотные притягивали взор в 21 \% случаев, изображения «средней» пространственной частоты - в $25 \%$, более высокочастотные - в 23 \% предъявлений. Чтобы определить, являются ли обнаруженные различия статистически значимыми, мы воспользовались все тем же G-критерием (см. таблицу 4).

Модуляции ориентации разной частоты, как и модуляции контраста, также демонстрировали статистически значимые различия в способности притягивать внимание испытуемых. Причем первый перевод взора чаще направлялся на изображения «средней» пространственной частоты. Однако, в отличие от модуляций контраста, реже других привлекали внимание самые низкочастотные изображения.

Таблица 4. Результаты статистического сравнения вероятностей первой фиксации взора на изображениях разной пространственной частоты, сформированных из моАуляций ориентации

Table 4. Results of statistical comparing the probabilities of participants' first fixations on the images composed of the modulations of orientation differing in their spatial frequency

\begin{tabular}{|c|c|c|}
\hline $\begin{array}{l}\text { Пары сравнения } \\
\text { Comparing pairs }\end{array}$ & $\begin{array}{l}\text { G-критерий } \\
\text { G-criterion }\end{array}$ & $\begin{array}{c}\text { р-значение } \\
\text { (Авустороннее) } \\
\text { p-value (two-tailed) }\end{array}$ \\
\hline $\begin{array}{l}8 \text { цикл./изобр. - } 16 \text { цикл./изобр. } \\
8 \text { суcles/image - } 16 \text { cycles/image }\end{array}$ & 8,0509 & 0,0045 \\
\hline $\begin{array}{l}8 \text { цикл./изобр. - } 32 \text { цикл./изобр. } \\
8 \text { cycles/image - } 32 \text { cycles/image }\end{array}$ & 1,7428 & 0,1868 \\
\hline $\begin{array}{l}16 \text { цикл./изобр. - } 32 \text { цикл./изобр. } \\
16 \text { cycles/image - } 32 \text { cycles/image }\end{array}$ & 2,3044 & 0,1290 \\
\hline
\end{tabular}




\section{Серия 3.}

В третьей серии экспериментов изображения были сформированы из модуляций пространственной частоты. Эти изображения отличались базовой пространственной частотой несущей (2, 4 и 8 цикл./град.), т. е. частотой, относительно которой имела место пространственная модуляция. Были получены следующие результаты.

Не было зарегистрировано ни одного случая направления первой фиксации взора на изображения с самой низкой базовой частотой. Это связано с тем, что на данной частоте искомые модуляции имеют очень низкую (часто подпороговую) амплитуду.

Из двух оставшихся изображений значительно чаще внимание привлекало то, базовая частота которого была ниже (в нашей градации «средней»). Это происходило в $36 \%$ случаев. Самые высокочастотные из использованных изображений привлекали внимание в 22 \% случаев. Результаты статистического сравнения полученных величин приведены в таблице 5. Поскольку не имело смысла включать в статистический анализ результат, равный нулю (отсутствие фиксаций на изображениях самой низкой частоты), мы сравнили лишь показатели, отличные от нуля.

Таблица 5. Результаты статистического сравнения вероятностей первой фриксации взора на изображениях разной пространственной частоты, сфоормированные из модуляций пространственной частоты

Table 5. Results of statistical comparing the probabilities of participants' first fixation on the images composed of the modulations of spatial frequency differing in their spatial frequency

\begin{tabular}{|c|c|c|}
\hline $\begin{array}{c}\text { Пары сравнения } \\
\text { Comparing pairs }\end{array}$ & G-критерий & $\begin{array}{c}\text { p-значение } \\
\text { (Авустороннее) }\end{array}$ \\
\hline 8-criterion & p-value (two-tailed) \\
\hline 8/32 цикл./изобр. - 16/64 цикл./изобр. & 65,9156 & $\mathbf{0 , 0 0 0 0}$ \\
\hline
\end{tabular}

Полученный результат указывает на сохранение все той же закономерности: более приоритетными для внимания являются модуляции, имеющие среднюю базовую пространственную частоту.

Таким образом, результаты второго эксперимента демонстрируют сходную закономерность для модуляций всех размерностей: более конкурентоспособными за внимание в рамках одной размерности оказались изображения, сформированные из «средних» пространственных частот. 


\section{Обсуждение результатов}

Совершенно очевидно, что пространственные неоднородности привлекают зрительное внимание. Взор в первую очередь будет направлен на границу между текстурами, а не на однородную область [26, 27]. Оказалось, что локализации фиксаций взора при рассматривании естественных изображений значительно лучше предсказываются моделью, когда карты целеуказаний (saliency maps) строятся с учетом пространственных модуляций градиентов яркости $[28,29,30]$. Однако возникает следующий вопрос: как сами модуляции конкурируют за внимание, какие пространственные неоднородности более приоритетны для привлечения внимания?

Мы попытались ответить на этот вопрос в эксперименте 1. Оказалось, что ни одна из размерностей модуляции не имеет абсолютного преимущества перед другими: за внимание конкурируют все. Но в то же время они не равноценны в этой конкуренции. Модуляции пространственной частоты статистически значимо уступают двум другим. Чем это можно объяснить?

Самым простым объяснением представляется следующее: из трех конкурирующих изображений первый перевод взора с большей вероятностью будет направляться на то, которое имеет более высокую энергию (большую спектральную мощность). Мы сравнили усредненные спектры изображений, сформированных из модуляций контраста, ориентации и пространственной частоты (усреднялось по 100 спектров для каждой размерности модуляции). Результат показан на рисунке 3.

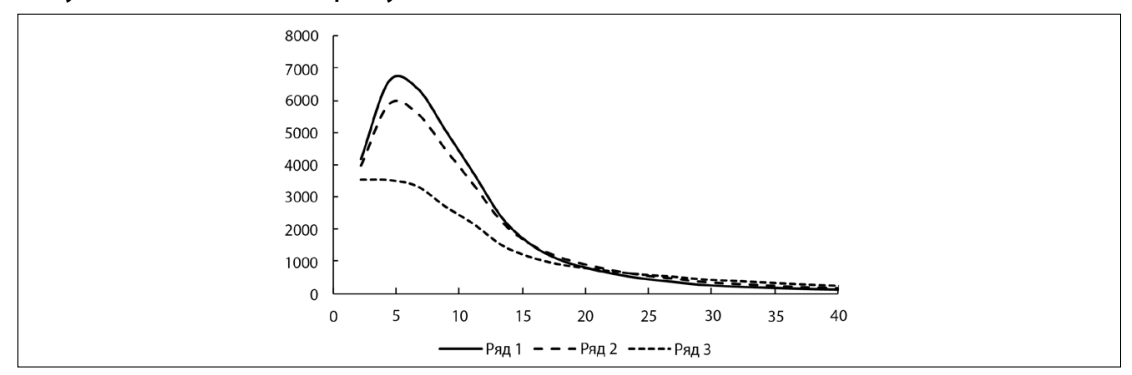

Рисунок 3. Средняя спектральная мощность изображений, сорормированных из модуляций контраста (ряА 1), ориентации (ряА 2) и пространственной частоты (ряА 3)

Условные обозначения: по оси абсцисс - пространственная частота (в чикл./изобр.), по оси ординат - мощность сигнала (в усл. ед.).

Figure 3. Average spectral power of images constructed from the modulations of contrast (row 1), orientation (row 2), and spatial frequency (row 3)

Legend: the abscissa shows spatial frequency (cycles/image); the ordinate shows signal strength (conventional units). 
Приведенный график подтверждает сделанное предположение: большую энергию имеют изображения, сформированные из областей с модуляциями контраста или ориентации. Изображения, сформированные из областей с модуляциями пространственной частоты, обладают существенно меньшей спектральной мощностью.

Чтобы еще раз проверить данное предположение, мы воспользовались результатами второго эксперимента. Следовало ожидать, что среди изображений одной размерности модуляции, но разной пространственной частоты, целью первой фиксации чаще должны быть те, которые имеют большую спектральную мощность. Мы вновь усреднили спектры всех восстановленных изображений, теперь в пределах определенной модуляции и пространственной частоты. Результат показан на рисунке 4.

Напомним, что во втором эксперименте первый перевод взора чаще направлялся на изображения, сформированные из «средних» пространственных частот. Однако приведенные графики подтверждают эту закономерность только для модуляций пространственной частоты (рисунок 4В), и то лишь потому, что модуляции данной размерности фактически отсутствуют в самом низкочастотном диапазоне. Таким образом, предположение, что приоритетными для внимания являются изображения с большей спектральной мощностью, не нашло подтверждения.

Однако возможно еще одно объяснение полученным результатам. Очевидно, что для управления вниманием «снизу» значение имеет не то, как распределен сигнал на входе зрительной системы, а то, как распределена нейронная активность на выходе преаттентивной стадии. В данном случае речь идет о «карте» пространственных модуляций. Приоритетной для внимания должна быть та область карты, которая характеризуется наибольшей активностью фильтров второго порядка. Данное предположение может быть проверено с использованием компьютерной модели этих фильтров. Одним из результатов работы этой модели как раз и является построение карты активности на выходах фильтров второго порядка (см. приложение).

Сначала обратимся к результатам второго эксперимента. Воспользовавшись расчетами модели, мы вычислили средний выход фильтров второго порядка для каждого восстановленного изображения, а затем полученные значения усреднили по 100 изображениям определенной размерности и пространственной частоты. Полученный результат показан на рисунке 5 .

Гистограммы свидетельствуют о том, что для всех модуляций наибольший выход (в среднем) имеют фильтры второго порядка, настроенные на «средние» пространственные частоты. Напомним, что в эксперименте 2 первый перевод взора чаще всего направлялся именно на те изображения, которые были составлены из модуляций «средней» пространственной частоты. 

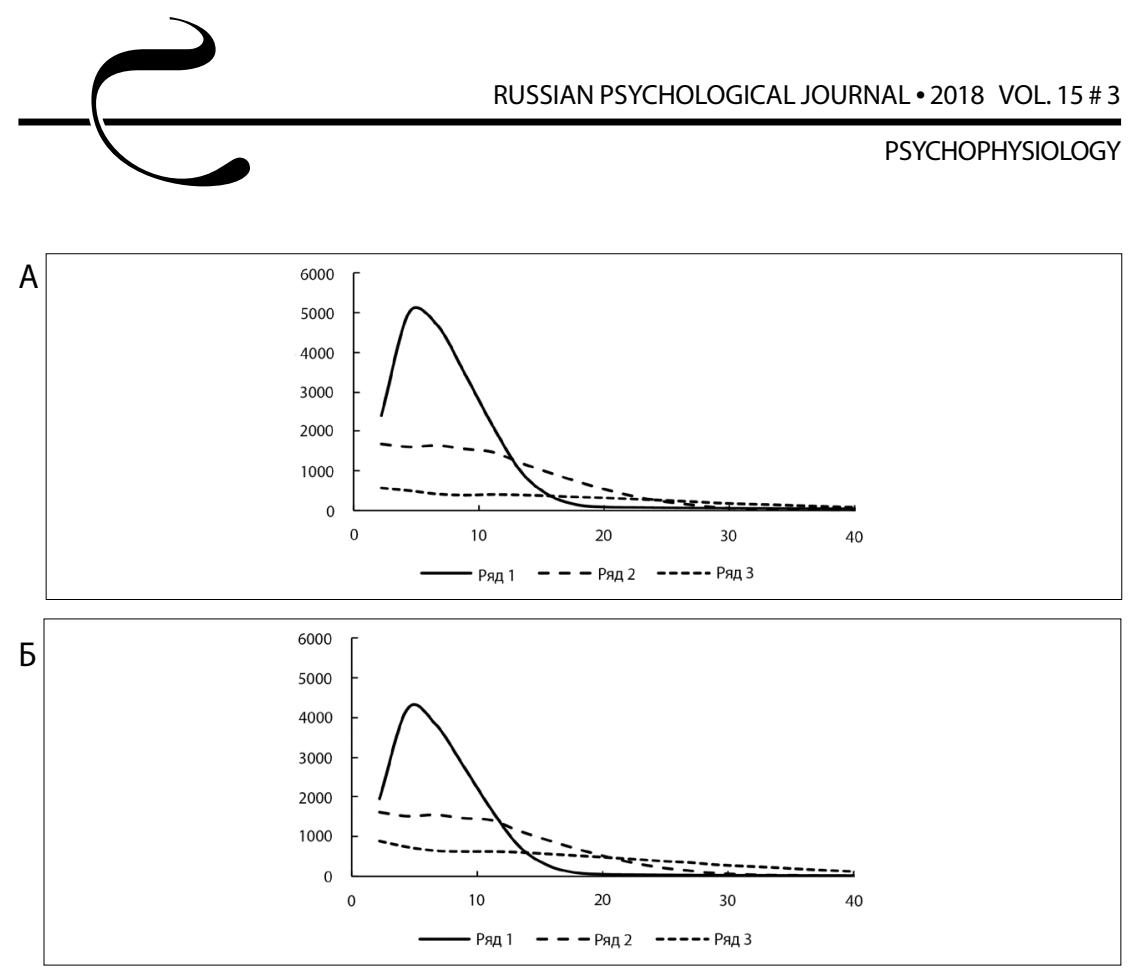

B

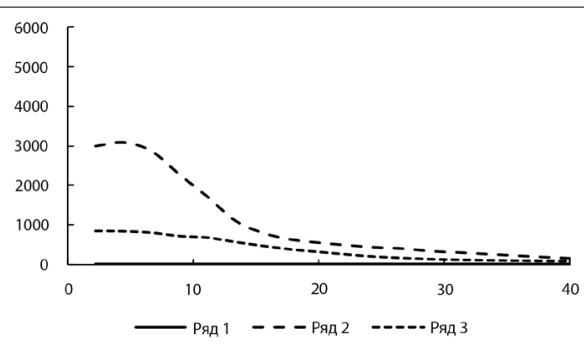

Рисунок 4. Средняя спектральная мощность изображений разной пространственной частоты, сорормированных из модуляций контраста (А), ориентации (Б) и пространственной частоты (В)

Условные обозначения: ряд 1 -спектры мощности изображений с пространственной частотой 8 чикл./изобр.; ряд 2 - 16 чикл./изобр.; ряд 3 - 32 чикл./изобр. (для модуляций пространственной частоты, соответственно, 4/16, 8/32 и 16/64 чикл./изобр.). По оси абсцисс- пространственная частота (цикл./изоб.), по оси ординат - мощность сигнала (в усл. ед.).

Figure 4. Average spectral power of images constructed from the modulations of contrast (A), orientation (B), and spatial frequency (C) differing in their spatial frequency

Legend: row 1 - power spectrum of images in a spatial frequency of 8 cycles/image; row 2 - 16 cycles/image; row 3 - 32 cycles/image (for the modulation of spatial frequency-4/16,8/32, and 16/64 cycles/image respectively). The abscissa shows spatial frequency (cycles/image); the ordinate shows signal strength (conventional units). 

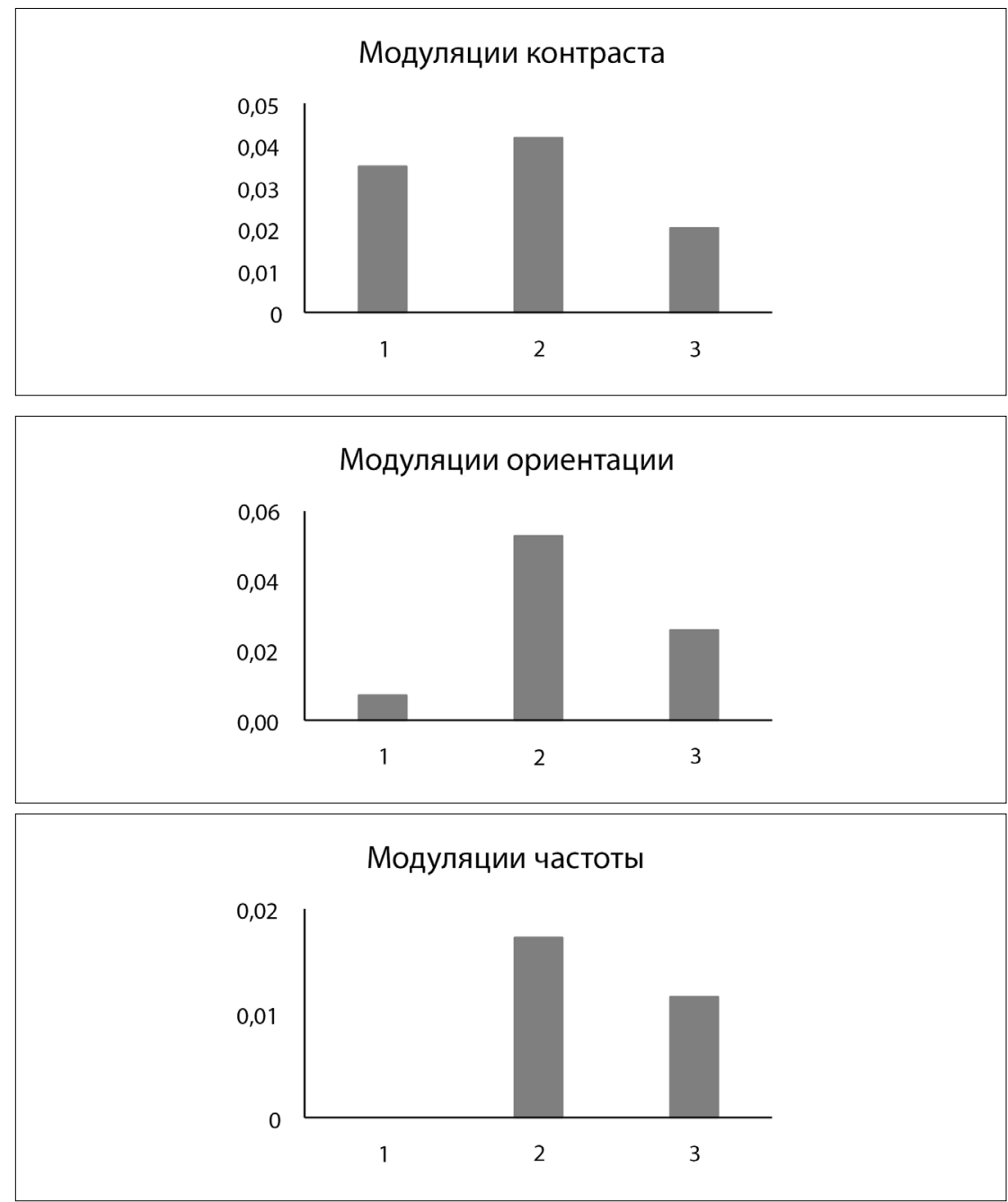

Рисунок 5. Среаний выход (нормализованный) фрильтров первого, второго и третьего слоя модели, участвующих в фрормировании тестовых изображений разной пространственной частоты

Figure 5. Average rates (normalized) of output signals in the first-, second-, and third-order filters involved in constructing test images differing in their spatial frequency 
Теперь вернемся к результатам первого эксперимента. Мы вновь воспользовались расчетами модели и вычислили средний выход фильтров второго порядка для каждого восстановленного изображения. Затем мы усреднили полученные результаты внутри каждой размерности модуляции (рисунок 6).

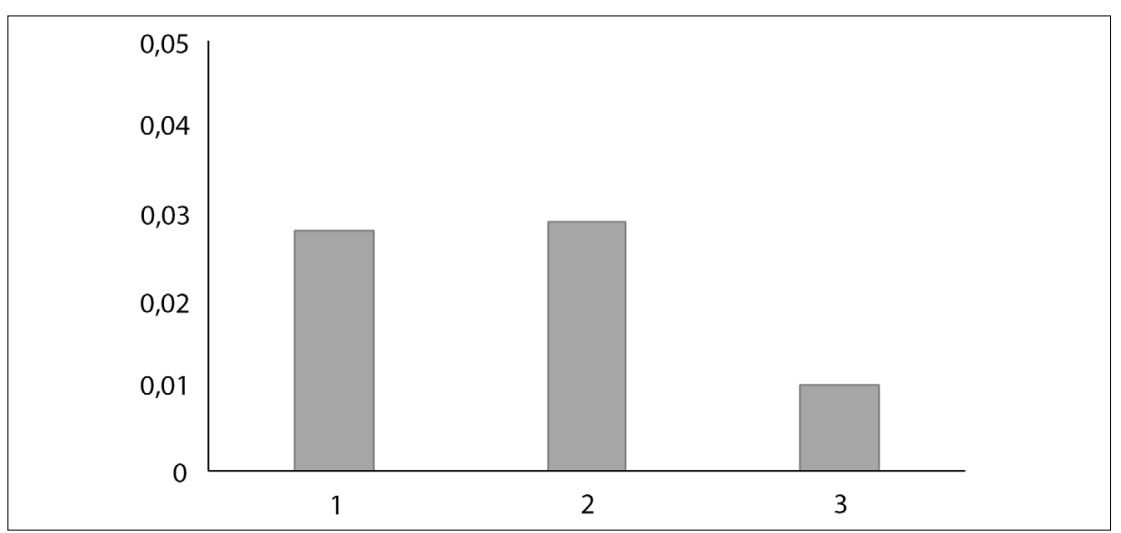

Рисунок 6. СреАний выхол Ф2П, формирующих тестовые изображения из разных моАуляций

Условные обозначения: 1 - модуляции контраста, 2- ориентации, 3-пространственной частоты.

Figure 6. Average rates of second-order filters output signal constructing test images from different modulations

Legend: 1 - modulations of contrast, 2 - orientation, and 3-spatial frequency.

График подтверждает правильность высказанного предположения. В среднем больший выход имеют фильтры второго порядка, избирательные к модуляциям контраста или ориентации. Изображения, сформированные именно из этих модуляций, чаще становились целью первой фиксации, чем изображения, собранные из модуляций пространственной частоты.

Итак, результаты обоих экспериментов согласуются с предположением, что внимание привлекается к тем областям поля зрения, которым соответствует больший средний уровень активности нейронов, выполняющих функцию фильтров второго порядка. Уровень же активности этих элементов пропорционален амплитуде выделенной ими модуляции. Таким образом, в конкуренции за внимание приоритет имеют области поля зрения, в которых более выражены пространственные изменения градиентов яркости.

Если перейти от искусственных условий нашего эксперимента к реальной ситуации, то картина может быть следующей. Когда в поле зрения появляется несколько разных объектов, внимание с большей вероятностью сначала 
будет направляться на тот объект, который содержит большие по амплитуде модуляции градиентов яркости (независимо от того, что изменяется: контраст, ориентация или пространственная частота). Внутри самого объекта области интереса также будут определяться наличием наиболее выраженных пространственных изменений. При этом с большей вероятностью это будут участки, в которых находятся модуляции контраста или ориентации в области средних пространственных частот, поскольку оказалось, что именно эти модуляции чаще имеют более высокую амплитуду в изображениях реальных объектов.

\section{Заключение}

Полученные результаты позволяют сделать следующие выводы:

1. Среди пространственных неоднородностей целью внимания чаще становятся модуляции контраста и ориентации в диапазоне средних пространственных частот.

2. Физиологическим механизмом управления вниманием «снизу» может быть распределение активности среди фильтров второго порядка. «Воротами» внимания с большей вероятностью становятся фильтры, имеющие более высокую активность.

3. Внимание привлекают области поля зрения с наибольшей амплитудой пространственных изменений параметров градиентов яркости.

\section{Приложение}

Многочисленные исследования [17] свидетельствуют о том, что преаттентивный этап зрительной обработки включает две стадии: выделение и кодирование отдельных градиентов яркости стриарными нейронами, а также пространственное объединение этой локальной информации экстрастриарными нейронами. В результате на выходе преаттентивной обработки выделяются области изображения, содержащие пространственные неоднородности: модуляции контраста, ориентации или пространственной частоты градиентов яркости. Разработанная нами компьютерная модель реализует 4 базовые операции: фильтрацию 1-го порядка, выпрямление, фильтрацию 2-го порядка и сегментацию входного изображения. Две первые операции моделируют работу фильтров 1-го порядка, две последующие операции работу фильтров 2-го порядка.

На вход модели подается цифровое изображение - растр, представленный 256 градациями яркости и помещенный в массив $L(x, y)$, где $x=1, \ldots, n_{x} ; y=1, \ldots, n_{y}$. 
Фильтрация 1-го порядка.

Импульсная характеристика фильтра 1-го порядка (весовая функция Габора) описывается следующим выражением [31]:

$$
\begin{aligned}
& G_{\lambda \theta \phi \sigma \gamma}(x, y)=\exp \left(\frac{-x^{\prime 2}+\gamma^{2} y^{\prime 2}}{2 \sigma^{2}}\right) \cos \left(2 \pi \frac{x^{\prime}}{\lambda}+\varphi\right), \\
& x^{\prime}=x \cos \theta+y \sin \theta, \\
& y^{\prime}=-x \sin \theta+y \cos \theta,
\end{aligned}
$$

где

$\lambda$ - длина волны фильтра на пиковой частоте фильтрации;

$b$ - полоса пропускания фильтра по пространственной частоте;

$\theta$ - пиковая ориентация фильтра;

Y - полоса пропускания фильтра по ориентации;

$\varphi$ - фазовый сдвиг фильтра.

Поскольку

$$
\frac{\sigma}{\lambda}=\frac{1}{\pi} \sqrt{\frac{\ln 2}{2}} \cdot \frac{2^{b}+1}{2^{b}-1}
$$

параметр б может быть вычислен из $\lambda$ и $b$.

Настройки фильтров, последовательность выполнения операций фильтрации и их число задаются следующими управляющими параметрами:

- количеством пространственно-частотных каналов $n_{\lambda}$;

- числом фазово-избирательных каналов - $n_{\varphi}$;

- частотными настройками $\lambda(i)$ каждого канала, $i=0, \ldots, n_{\lambda}-1$;

- количеством ориентационно-избирательных каналов $n_{\theta}$;

- ориентационными настройками $\theta(j)$ каждого ориентационно-избирательного канала, $j=0, \ldots, n_{\theta}-1$.

На основании физиологических данных были приняты следующие значения управляющих параметров:

- $n_{\lambda}=6$ и $\lambda(i)=p \cdot 2^{i-1}$, где $i=0, \ldots, n_{\lambda}-1$, а $p$ - коэффициент перехода от условных единиц к пикселям;

- $n_{\theta}=6$ и $\theta(j)=q \cdot j$, где $j=0, \ldots, n_{\theta}-1$, а $q$ - коэффициент перехода к градусам $(q=30)$;

- $n_{\varphi}=2$ и $\varphi(k)=90 \cdot k$, где $k=0, \ldots, n_{\varphi}-1$;

$-b=2$;

$-\gamma=0,5$. 
Полосовая фильтрация входного изображения реализуется набором линейных фильтров с разной фазовой, ориентационной и пространственночастотной настройкой:

$$
E_{\lambda(i) \theta(j) \varphi(k)}(x, y)=L(x, y) * G_{\lambda(i) \theta(j) \varphi(k) \sigma \gamma}(x, y) .
$$

\section{Выпрямление.}

Значения выходов двух фильтров, имеющих одинаковые пространственночастотные и ориентационные, но разные фазовые настройки, рассчитываются как квадратные корни поэлементных сумм квадратов выходов этих фильтров:

$$
E_{\lambda(i) \theta(j)}(x, y)=\sqrt{\sum_{k=0}^{n_{\varphi}-1} E_{\lambda(i) \theta(j) \varphi(k)}(x, y)^{2}} .
$$

Результаты операций фильтрации и выпрямления записываются в массивы $E_{\lambda(i) \theta(j)}(x, y)$, которые являются входами для следующей операции фильтрации 2-го порядка. Общее число массивов равно $n_{\lambda} * n_{\theta}$ (по числу фильтров с разными пространственно-частотными настройками для каждой ориентации фильтра).

\section{Фильтрация 2-го порядка.}

Массивы $E_{\lambda(i) \theta(j)}(x, y)$ являются входом для фильтров 2-го порядка. В модели реализовано фиксированное отношение частотных настроек фильтров 1-го и 2-го порядка, задаваемое коэффициентом $R$.

На уровне фильтров 2-го порядка повторяется операция полосовой линейной фильтрации, которая описывается следующим выражением:

$$
S(x, y)=A(x, y)-I(x, y),
$$

где $A$ - свертка $E$ с возбудительной частью поля, $I$ - свертка $E$ с тормозной частью поля.

Свертка с возбудительной частью поля является общей для всех фильтров и описывается следующим выражением:

$$
A(x, y)=E_{\lambda(c) \theta(d)}(x, y) * G p_{(\lambda(c) \cdot R) \theta(d) \phi \sigma \gamma}(x, y),
$$

где $G p$ - весовая функция возбудительной части поля, причем $c \in I=\left\{i=0, \ldots, n_{\lambda}-1\right\}, d \in J=\left\{j=0, \ldots, n_{\theta}-1\right\}$.

Свертка с тормозной частью поля отличается для фильтров, избирательных к разной размерности модуляции. Именно организация тормозных флангов фильтра определяет его специфичность. 


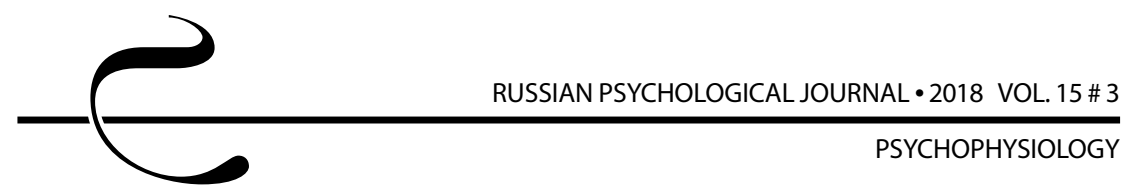

Для фильтров, избирательных к модуляциям контраста, свертка с тормозной частью поля описывается следующим выражением:

$$
I(x, y)=\max \left(E_{\lambda(I \backslash\{c\}) \theta(J \backslash\{d\})}(x, y)\right) * G n_{(\lambda(c) \cdot R) \theta(d) \phi \sigma \gamma}(x, y),
$$

где $\backslash$ - оператор исключения из множества, max - поэлементный оператор выбора максимального из соответственных значений нескольких матриц с согласованными размерами, Gn - весовая функция тормозной части поля.

Избирательность фильтра к модуляциям контраста достигается тем, что его тормозные фланги сформированы выходами фильтров 1-го порядка во всем диапазоне ориентационных и частотных настроек.

Для фильтров, избирательных к модуляциям ориентации, свертка с тормозной частью поля описывается следующим выражением:

$$
I(x, y)=\left(K \cdot \operatorname{summ}\left(E_{\lambda(I) \theta(d)}(x, y)\right)\right) * G n_{(\lambda(c) \cdot R) \theta(d) \phi \sigma \gamma}(x, y),
$$

где $K$ - весовой коэффициент тормозного входа, sитm - оператор сложения матриц.

Избирательность фильтра к модуляциям ориентации обеспечивается объединением в тормозных флангах выходов фильтров 1-го порядка во всем диапазоне частотных настроек.

Для фильтров, избирательных к модуляциям пространственной частоты, свертка с тормозной частью поля описывается следующим выражением:

$$
I(x, y)=\left(K \cdot \operatorname{summ}\left(E_{\lambda(c) \theta(J)}(x, y)\right)\right) * G n_{(\lambda(c) \cdot R) \theta(d) \phi \sigma \gamma}(x, y) .
$$

Избирательность фильтра к модуляциям пространственной частоты достигается благодаря тому, что тормозные фланги сформированы выходами фильтров 1-го порядка во всем диапазоне ориентационных настроек.

Для фильтров, избирательных к модуляциям ориентации и пространственной частоты, дополнительно вводится операция нормирования входа $E_{\lambda(c) \theta(J)}(x, y)$ относительно результатов его фильтрации фильтром Гаусса $(\sigma=2 \cdot \lambda(c))$. Это сделано для того, чтобы добиться их нечувствительности к модуляциям контраста. Результатами выполнения операций свертки для каждой длины волны и ориентации фильтра будут $n_{\theta}$ массивов $I_{(\lambda(c) \cdot R) \theta(d)}(x, y)$. Результаты фильтрации на каждой частоте объединяются между ориентациями при помощи операции тах:

$$
I_{(\lambda(c) \cdot R)}(x, y)=\max \left(I_{(\lambda(c) \cdot R) \theta(J)}(x, y)\right) .
$$

\section{Сегментация.}

Завершающей операций работы модели является построение карты потенциальных целей внимания. Для этого выполняются следующие действия: 
1. Латеральное торможение между выходами фильтров 2-го порядка реализуется операцией фильтрации каждого $I_{(\lambda(c) \cdot R)}(x, y)$ с маской, предложенной Х. Дуанем с соавт. [32]:

$\begin{array}{rcccc}0,025 & 0,025 & 0,025 & 0,025 & 0,025 \\ 0,025 & 0,075 & 0,075 & 0,075 & 0,025 \\ 0,025 & 0,075 & 1 & 0,075 & 0,025 . \\ 0,025 & 0,075 & 0,075 & 0,075 & 0,025 \\ 0,025 & 0,025 & 0,025 & 0,025 & 0,025\end{array}$

2. В каждом массиве $I_{(\lambda(c) \cdot R)}(x, y)$ находятся локальные максимумы фильтры 2-го порядка с наибольшим выходом по сравнению с соседями.

Эти фильтры являются потенциальными воротами внимания.

\section{Благодарности}

Исследование выполнено при финансовой поддержке РФФИ (№ 17-06-50141-ОГН\18).

\section{Acknowledgments}

The study received funding through the Russian Foundation for Basic Research (project no. 17-06-50141).

\section{Литература}

1. Ярбус А. Л. Роль движений глаз в процессе зрения. М: Наука, 1965. 167 с.

2. Borji A., Itti L. State-of-the-Art in Visual Attention Modeling // IEEE Transactions on Pattern Analysis and Machine Intelligence. 2013. Vol. 35, Issue 1. P. 185-207. DOI: $10.1109 /$ tpami.2012.89

3. Шурупова М. А., Анисимов В. Н., Терещенко Л. В., Латанов А. В. Влияние когнитивной задачи на параметры движений глаз при просмотре статических и динамических сцен // Сенсорные системы. 2016. Т. 30, № 1. C. 53-62.

4. Zhang B., Liu S., Doro M., Galfano G. Attentional Guidance from Multiple Working Memory Representations: Evidence from Eye Movements // Scientific Reports. 2018. Vol. 8, Article number 13876.DOI: 10.1038/s41598-018-32144-4

5. Henderson J. M., Weeks P. A. Jr., Hollingworth A. The effects of semantic consistency on eye movements during complex scene viewing // Journal of Experimental Psychology: Human Perception and Performance. 1999. Vol. 25, № 1. P. 210-228. DOI: 10.1037/0096-1523.25.1.210

6. Orquin J. L., Loose S. M. Attention and choice: a review on eye movements in decision making // Acta Psychologica. 2013. Vol. 144, Issue 1. P. 190-206. DOI: $10.1016 /$ j.actpsy.2013.06.003 
7. Theeuwes J. Visual Selection: Usually Fast and Automatic; Seldom Slow and Volitional; A Reply to Commentaries // Journal of Cognition. 2018. Vol. 1 (1). P. 21. DOI: $10.5334 /$ joc.32

8. Treisman A. M., Gelade G. A feature-integration theory of attention // Cognitive Psychology. 1980. Vol. 12, Issue 1. P. 97-136. DOI: 10.1016/00100285(80)90005-5

9. Eimer $M$. The neural basis of attentional control in visual search // Trends in Cognitive Sciences. 2014. Vol. 18, Issue 10. P. 526-535. DOI: 10.1016/j. tics.2014.05.005

10. Wolfe J. M., Cave K. R., Franzel S. L. Guided search: an alternative to the feature integration model for visual search // Journal of Experimental Psychology: Human Perception and Performance. 1989. Vol. 15, № 3. P. 419-433.

11. Becker S. I., Harris A. M., Venini D. R., James D. Visual search for color and shape: When is the gaze guided by feature relationships, when by feature values? // Journal of Experimental Psychology: Human Perception and Performance. 2014. Vol. 40, № 1. P. 264-291. DOI: $10.1037 / a 0033489$

12. Cajar A., Engbert R., Laubrock J. Spatial frequency processing in the central and peripheral visual field during scene viewing // Vision Research. 2016. Vol. 127. P. 186-197. DOI: $10.1016 /$ j.visres.2016.05.008

13. Wolfe J. M. Guided search 4.0. Current Progress With a Model of Visual Search // Integrated Models of Cognitive Systems / W. D. Gray (Ed.). Oxford University Press, 2007. P. 99-156. DOI: 10.1093/acprof:oso/9780195189193.001.0001

14. Canosa R. L., Pelz J. B., Mennie N. R., Peak J. High-level aspects of oculomotor control during viewing of natural-task images // Proceedings Volume 5007 "Human vision and electronic imaging VIII" / B. E. Rogowitz, T. N. Pappas (Eds.). Santa Clara, California, USA, 2003. DOI: $10.1117 / 12.477375$

15. Henderson J. M., Hayes T. R., Rehrig G., Ferreira F. Meaning Guides Attention during Real-World Scene Description // Scientific Reports. 2018. Vol. 8, Article number 13504. DOI: 10.1038/s41598-018-31894-5

16. Wang W., Shen J. Deep Visual Attention Prediction // IEEE Transactions on Image Processing. 2018. Vol. 27, Issue 5. P. 2368-2378. DOI: 10.1109/TIP.2017.2787612

17. Graham N. V. Beyond multiple pattern analyzers modeled as linear filters (as classical V1 simple cells): Useful additions of the last 25 years // Vision Research. 2011. Vol. 51, Issue 13. P. 1397-1430. DOI: 10.1016/j.visres.2011.02.007

18. Dakin S. C., Mareschal I. Sensitivity to contrast modulation depends on carrier spatial frequency and orientation // Vision Research. 2000. Vol. 40, Issue 3. P. 311-329. DOI: 10.1016/S0042-6989(99)00179-0

19. Landy M. S., Oruç I. Properties of second-order spatial frequency channels // Vision Research. 2002. Vol. 42, Issue 19. P. 2311-2329. DOI: $10.1016 /$ S00426989(02)00193-1 
20. Явна Д. В., Куприянов И. В., Кокорникова В.И. Отражение процессов восприятия модуляций ориентации в вызванных ответах мозга человека // Российский психологический журнал. 2015. Т. 12, № 4. С. 161-274. DOI: 10.21702/rpj.2015.4.13

21. Hallum L. E., Movshon J. A. Surround suppression supports second-order feature encoding by macaque V1 and V2 neurons // Vision Research. 2014. Vol. 104. P. 24-35. DOI: $10.1016 /$ j.visres.2014.10.004

22. Барабанщиков В. А., Жегалло А. В. Регистрация и анализ направленности взора человека. М: Изд-во Института психологии РАН, 2013. 316 с.

23. Theeuwes J. Top-down and bottom-up control of visual selection // Acta Psychologica. 2010. Vol. 135, Issue 2. P. 77-99. DOI: $10.1016 /$ j.actpsy.2010.02.006

24. Wilson H. R., Gelb D. J. Modified line-element theory for spatial-frequency and width discrimination // Journal of the Optical Society of America A. 1984. Vol. 1, Issue 1. P. 124-131. DOI: $10.1364 /$ JOSAA.1.000124

25. McDonald J. H. Handbook of Biological Statistics (3rd ed.). Baltimore: Sparky House Publishing, 2014. 305 p.

26. Nothdurft $H$. C. Texture segmentation and pop-out from orientation contrast // Vision Research. 1991. Vol. 31, Issue 6. P. 1073-1078. DOI: 10.1016/0042-6989(91)90211-M

27. Schmid A. M., Victor J. D. Possible functions of contextual modulations and receptive field nonlinearities: Pop-out and texture segmentation // Vision Research. 2014. Vol. 104. P. 57-67. DOI: 10.1016/j.visres.2014.07.002

28. Johnson A., Zarei A. Second-order saliency predicts observer eye movements when viewing natural images // Journal of Vision. 2010. Vol. 10, Issue 7. P. 526. DOI: $10.1167 / 10.7 .526$

29. Cheng M.-M., Mitra N. J., Huang X., Torr P. H. S., Hu S.-M. Global Contrast Based Salient Region Detection // IEEE Transactions on Pattern Analysis and Machine Intelligence. 2015. Vol. 37, Issue 3. P. 569-582. DOI: $10.1109 /$ tpami.2014.2345401

30. Onat S., Açık A., Schumann F., König P. The Contributions of Image Content and Behavioral Relevancy to Overt Attention // PLoS ONE. Vol. (4). e93254. DOI: $10.1371 /$ journal.pone.0093254

31. Grigorescu C., Petkov N., Westenberg M. A. Contour detection based on nonclassical receptive field inhibition // IEEE Transactions on Image Processing. 2003. Vol. 12, Issue 7. P. 729-739. DOI: $10.1109 /$ TIP.2003.814250

32. Duan H., Deng Y., Wang X., Xu C. Small and Dim Target Detection via Lateral Inhibition Filtering and Artificial Bee Colony Based Selective Visual Attention // PLoS ONE. 2013. Vol. 8, № 8. e72035. DOI: 10.1371/journal.pone.0072035 


\section{References}

1. Yarbus A. L. Rol'dvizhenii glaz v protsesse zreniya [Eye movements and vision]. Moscow, Nauka Publ., 1965. 167 p.

2. Borji A., Itti L. State-of-the-art in visual attention modeling. IEEE Transactions on Pattern Analysis and Machine Intelligence, 2013, V. 35, Issue 1, pp. 185-207. DOI: 10.1109/tpami.2012.89

3. Shurupova M. A., Anisimov V. N., Tereshchenko L. V., Latanov A. V. Impact of cognitive tasks on the parameters of eye movements when viewing static and dynamic scenes. Sensornye sistemy - Sensory Systems, 2016, V. 30, no. 1, pp. 53-62 (in Russian).

4. Zhang B., Liu S., Doro M., Galfano G. Attentional guidance from multiple working memory representations: Evidence from eye movements. Scientific Reports, 2018, V. 8, Article number 13876. DOI: 10.1038/s41598018-32144-4

5. Henderson J. M., Weeks P. A. Jr., Hollingworth A. The effects of semantic consistency on eye movements during complex scene viewing. Journal of Experimental Psychology: Human Perception and Performance, 1999, V. 25, no. 1, pp. 210-228. DOI: 10.1037/0096-1523.25.1.210

6. Orquin J. L., Loose S. M. Attention and choice: a review on eye movements in decision making. Acta Psychologica, 2013, V. 144, Issue 1, pp. 190-206. DOI: $10.1016 /$ j.actpsy.2013.06.003

7. Theeuwes J. Visual selection: Usually fast and automatic; Seldom slow and volitional; A reply to commentaries. Journal of Cognition, 2018, V. 1 (1), p. 21. DOI: $10.5334 /$ joc.32

8. Treisman A. M., Gelade G. A feature-integration theory of attention. Cognitive Psychology, 1980, V. 12, Issue 1, pp. 97-136. DOI: 10.1016/00100285(80)90005-5

9. Eimer M. The neural basis of attentional control in visual search. Trends in Cognitive Sciences, 2014, V. 18, Issue 10, pp. 526-535. DOI: 10.1016/j. tics.2014.05.005

10. Wolfe J. M., Cave K. R., Franzel S. L. Guided search: an alternative to the feature integration model for visual search. Journal of Experimental Psychology: Human Perception and Performance, 1989, V. 15, no. 3, pp. 419-433.

11. Becker S. I., Harris A. M., Venini D. R., James D. Visual search for color and shape: When is the gaze guided by feature relationships, when by feature values? Journal of Experimental Psychology: Human Perception and Performance, 2014, V. 40, no. 1, pp. 264-291. DOI: 10.1037/a0033489

12. Cajar A., Engbert R., Laubrock J. Spatial frequency processing in the central and peripheral visual field during scene viewing. Vision Research, 2016, V. 127, pp. 186-197. DOI: $\underline{10.1016 / \text { j.visres.2016.05.008 }}$ 
13. Wolfe J. M. Guided search 4.0. Current progress with a model of visual search. In:W. D. Gray (ed.) Integrated Models of Cognitive Systems. Oxford University Press, 2007, pp. 99-156. DOI: 10.1093/acprof:oso/9780195189193.001.0001

14. Canosa R. L., Pelz J. B., Mennie N. R., Peak J. High-level aspects of oculomotor control during viewing of natural-task images. In: B. E. Rogowitz, T. N. Pappas (eds.) Proceedings Volume 5007 "Human vision and electronic imaging VIII". Santa Clara, California, USA, 2003. DOI: $10.1117 / 12.477375$

15. Henderson J. M., Hayes T. R., Rehrig G., Ferreira F. Meaning guides attention during real-world scene description. Scientific Reports, 2018, V. 8, Article number 13504. DOI: 10.1038/s41598-018-31894-5

16. Wang W., Shen J. Deep Visual Attention Prediction. IEEE Transactions on Image Processing, 2018, V. 27, Issue 5, pp. 2368-2378. DOI: $10.1109 /$ TIP.2017.2787612

17. Graham N. V. Beyond multiple pattern analyzers modeled as linear filters (as classical V1 simple cells): Useful additions of the last 25 years. Vision Research, 2011, V. 51, Issue 13, pp. 1397-1430. DOI: 10.1016/j.visres.2011.02.007

18. Dakin S. C., Mareschal I. Sensitivity to contrast modulation depends on carrier spatial frequency and orientation. Vision Research, 2000, V. 40, Issue 3, pp. 311-329. DOI: 10.1016/S0042-6989(99)00179-0

19. Landy M. S., Oruç I. Properties of second-order spatial frequency channels. Vision Research, 2002, V. 42, Issue 19, pp. 2311-2329. DOI: 10.1016/S00426989(02)00193-1

20. Yavna D. V., Kupriyanov I. V., Kokornikova V. I. The perception of the orientationally modulated textures and its expression in the visual evoked potentials. Rossiiskii psikhologicheskii zhurnal - Russian Psychological Journal, 2015, V. 12, no. 4, pp. 161-274 (in Russian). DOI: 10.21702/rpj.2015.4.13

21. Hallum L. E., Movshon J. A. Surround suppression supports second-order feature encoding by macaque V1 and V2 neurons. Vision Research, 2014, V. 104, pp. 24-35. DOI: 10.1016/j.visres.2014.10.004

22. Barabanshchikov V. A., Zhegallo A. V. Registratsiya i analiz napravlennosti vzora cheloveka [Registration and analysis of human eye orientation]. Moscow, Institute of Psychology RAS, 2013. 316 p.

23. Theeuwes J. Top-down and bottom-up control of visual selection. Acta Psychologica, 2010, V. 135, Issue 2, pp. 77-99. DOI: 10.1016/j. actpsy.2010.02.006

24. Wilson H. R., Gelb D. J. Modified line-element theory for spatial-frequency and width discrimination. Journal of the Optical Society of America, 1984, V. 1, Issue 1, pp. 124-131. DOI: 10.1364/JOSAA.1.000124

25. McDonald J. H. Handbook of biological statistics (3rd ed.). Baltimore, Sparky House Publishing, 2014. 305 p. 
26. Nothdurft H. C. Texture segmentation and pop-out from orientation contrast. Vision Research, 1991, V. 31, Issue 6, pp. 1073-1078. DOI: 10.1016/00426989(91)90211-M

27. Schmid A. M., Victor J. D. Possible functions of contextual modulations and receptive field nonlinearities: Pop-out and texture segmentation. Vision Research, 2014, V. 104, pp. 57-67. DOI: 10.1016/j.visres.2014.07.002

28. Johnson A., Zarei A. Second-order saliency predicts observer eye movements when viewing natural images. Journal of Vision, 2010, V. 10, Issue 7, p. 526. DOI: $10.1167 / 10.7 .526$

29. Cheng M.-M., Mitra N. J., Huang X., Torr P. H. S., Hu S.-M. Global contrast based salient region detection. IEEE Transactions on Pattern Analysis and Machine Intelligence, 2015, V. 37, Issue 3, pp. 569-582. DOI: 10.1109/ tpami.2014.2345401

30. Onat S., Açık A., Schumann F., König P. The contributions of image content and behavioral relevancy to overt attention. PLOS ONE, V. (4), e93254. DOI: 10.1371/journal.pone.0093254

31. Grigorescu C., Petkov N., Westenberg M. A. Contour detection based on nonclassical receptive field inhibition. IEEE Transactions on Image Processing, 2003, V. 12, Issue 7, pp. 729-739. DOI: $10.1109 /$ TIP.2003.814250

32. Duan H., Deng Y., Wang X., Xu C. Small and dim target detection via lateral inhibition filtering and artificial bee colony based selective visual attention. PLoS ONE, 2013, V. 8, no. 8, e72035. DOI: 10.1371/journal.pone.0072035 